\title{
Technè
}

La science au service de l'histoire de l'art et de la préservation des biens culturels

40 | 2014

Thérapéia. Polychromie et restauration de la sculpture dans l'Antiquité

\section{Peindre et repeindre sur terre cuite en Grèce hellénistique}

Painting and repainting terracotta figurines in Hellenistic Greece

\section{Brigitte Bourgeois et Violaine Jeammet}

\section{Q OpenEdition}

12 Journals

Édition électronique

URL : https://journals.openedition.org/techne/3762

DOI : 10.4000/techne.3762

ISSN : 2534-5168

Éditeur

C2RMF

\section{Édition imprimée}

Date de publication : 26 novembre 2014

Pagination : 84-95

ISBN : 978-2-7118-6218-4

ISSN : 1254-7867

Référence électronique

Brigitte Bourgeois et Violaine Jeammet, " Peindre et repeindre sur terre cuite en Grèce hellénistique », Technè [En ligne], 40 | 2014, mis en ligne le 24 juillet 2020, consulté le 08 février 2022. URL : http:// journals.openedition.org/techne/3762 ; DOI : https://doi.org/10.4000/techne.3762

\section{(c) (i) $\odot$}

La revue Technè. La science au service de l'histoire de l'art et de la préservation des biens culturels est mise à disposition selon les termes de la Licence Creative Commons Attribution - Pas d'Utilisation Commerciale - Pas de Modification 4.0 International. 
Brigitte Bourgeois

Violaine Jeammet

\section{Peindre et repeindre sur terre cuite en Grèce hellénistique}

Painting and repainting terracotta figurines in Hellenistic Greece

Résumé. Originellement peintes et/ou dorées, les figurines en terre cuite grecques ont parfois été repeintes avant d'être ensevelies ou détruites. L'article s'attache à cette question encore méconnue et présente les recherches menées sur deux statuettes d'époque hellénistique conservées au Louvre : une Ménade (dite Dame Baillehache) de production attique, et un Éros de Priène (Asie mineure). L'étude menée en vidéo-microscopie éclaire la stratigraphie des repeints et montre comment, dans le cas de la Ménade, la forme plastique accidentée a été "réparée » au moyen de la peinture; elle permet enfin de juger d'effets picturaux introduits aussi bien au stade initial que lors de la reprise de polychromie, visant à accentuer les jeux d'ombres et de lumières.

Mots-clés. Grèce, polychromie, coroplathie, terre cuite, repeint, examen, vidéo-microscopie, microscopie 3D de surface, Dame Baillehache, Éros de Priène, Louvre.

\section{Introduction}

À la différence du travail du marbre, l'artisanat grec de la terre cuite n'a quasiment pas laissé de trace dans les écrits antiques. Notre compréhension des modes et des techniques de production - qu'il s'agisse des étapes du façonnage ou de la finition colorée -comme de la fonction des objets repose donc essentiellement sur deux types de sources: $1^{\circ}$ ) les données du contexte archéologique, lorsque celui-ci est connu, $2^{\circ}$ ) l'étude approfondie de la matière, favorisée, si l'enquête porte sur les questions de polychromie, par la fréquente conservation de vestiges de peinture, de dorure, voire de vernis sur le support argileux. La terre cuite offre de ce point de vue un terrain d'observation privilégié, à la différence de la grande sculpture de marbre, lessivée par le temps et les hommes.

Cette qualité de conservation a permis de bonne heure à des savants, précurseurs en leur domaine, d'identifier deux phénomènes encore obscurs au milieu du $\mathrm{XX}^{\mathrm{e}}$ siècle: d'une part, l'influence croissante de la peinture sur les autres arts en Grèce, à partir du IV siècle av. J.-C., entraînant la diffusion de pratiques picturales dont les artisans de l'époque
Abstract. Originally painted and/or gilded, Greek terracotta figurines were sometimes repainted before being buried or destroyed. This article focuses on this still little-known question and presents the research conducted on two statuettes dating from the Hellenistic period now in the Louvre: a Maenad (known as Dame Baillehache) from Attica, and an Eros from Priene (Asia Minor). The study by video microscopy has shed light on the stratigraphy of the repaints and shown how, in the case of the Maenad, the damaged shape was "repaired" with paint. It also enabled us to assess the pictorial effects introduced in both the initial stage and when the polychromy was reworked in order to enhance the play of light and shadow.

Keywords. Greece, polychromy, coroplastics, terracotta, repaint, examination, video microscopy, 3D surface microscopy, Dame Baillehache, Priene Eros, Louvre.

hellénistique ont largement fait usage ${ }^{1}$; d'autre part, la présence, sur certaines figurines, de repeints antiques témoignant de l'attention portée à faire durer le chatoiement de la couleur. Dès 1931, Dorothy Burr avait relevé l'emploi de procédés picturaux sur certaines des meilleures productions de Myrina (Éolide, Turquie) : sur une statuette de Victoire datée du $\mathrm{II}^{\mathrm{e}}$ siècle av. J.-C., de fines touches de rouge ombraient les plis d'une tunique (chiton) blanche; ailleurs, la couleur était montée par superposition de couches picturales rose et rouge. Elle interprétait aussi le cas d'une Victoire comme un exemple de repeint ${ }^{2}$. Cependant, le manque d'illustration détaillée rendait et rend encore difficile d'apprécier pleinement sa démonstration.

Vingt-cinq ans plus tard, Alfred Laumonier publiait l'important corpus des terres cuites de Délos, l'île sainte au cœur des Cyclades, consacrée dans l'Antiquité à Apollon et Artémis ${ }^{3}$. Provenant de lieux publics, d'ateliers-boutiques et de riches maisons, le matériel datait pour l'essentiel du II $^{\mathrm{e}}$ siècle et du début du I ${ }^{\mathrm{er}}$ siècle av. J.-C., lorsque Délos, déclarée port franc par Rome, devint l'un des principaux carrefours commerciaux et culturels du monde hellénistique. Outre le nombre et la qualité de certaines pièces, le corpus délien offrait l'intérêt

Brigitte Bourgeois, conservateur en chef, chargée de mission sur l'histoire de la restauration, C2RMF (brigitte.bourgeois@culture.gouv.fr).

Violaine Jeammet, conservateur en chef, département des Antiquités grecques, étrusques et romaines, musée du Louvre (violaine.jeammet@louvre.fr). 


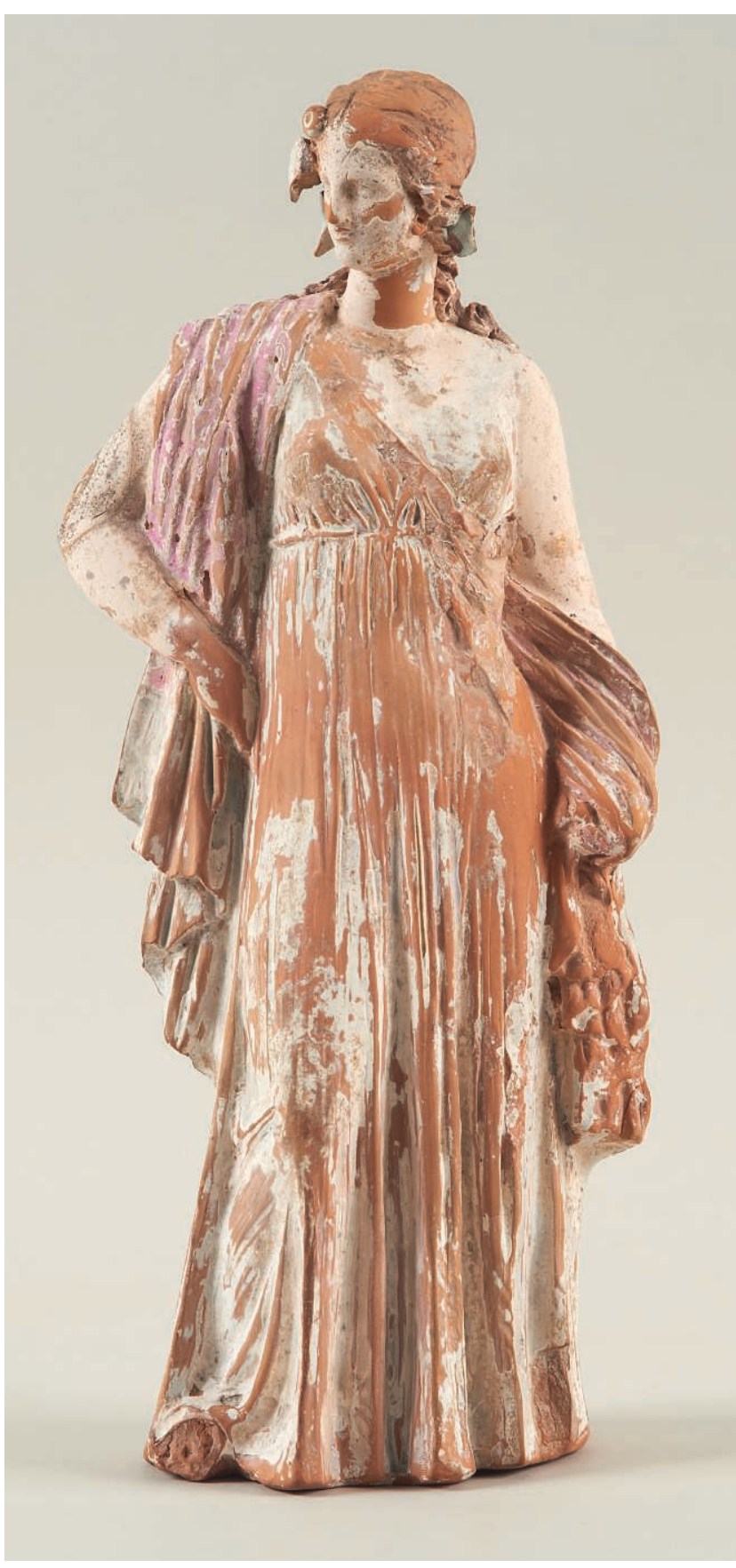

Fig. 1 a. Figurine de Ménade dite

Dame Baillehache, Attique, vers 330-200 av. J.-C.,

terre cuite peinte, H. 27,5 cm, Louvre, CA 2552.

Vue d'ensemble. (C) C2RMF/Anne Chauvet.

d'une collection demeurée exempte de repeints modernes. Observateur attentif, Laumonier sut détecter, sur certaines pièces, des repeints sous forme de strates de diverses couleurs, séparées par des couches successives de préparation. Ses remarques ont depuis lors été complétées par les travaux menés par Brigitte Bourgeois et Philippe Jockey sur la polychromie de la sculpture hellénistique de Délos ${ }^{4}$.

En dépit de ces débuts prometteurs, l'étude de la polychromie des terres cuites d'époque classique et hellénistique est

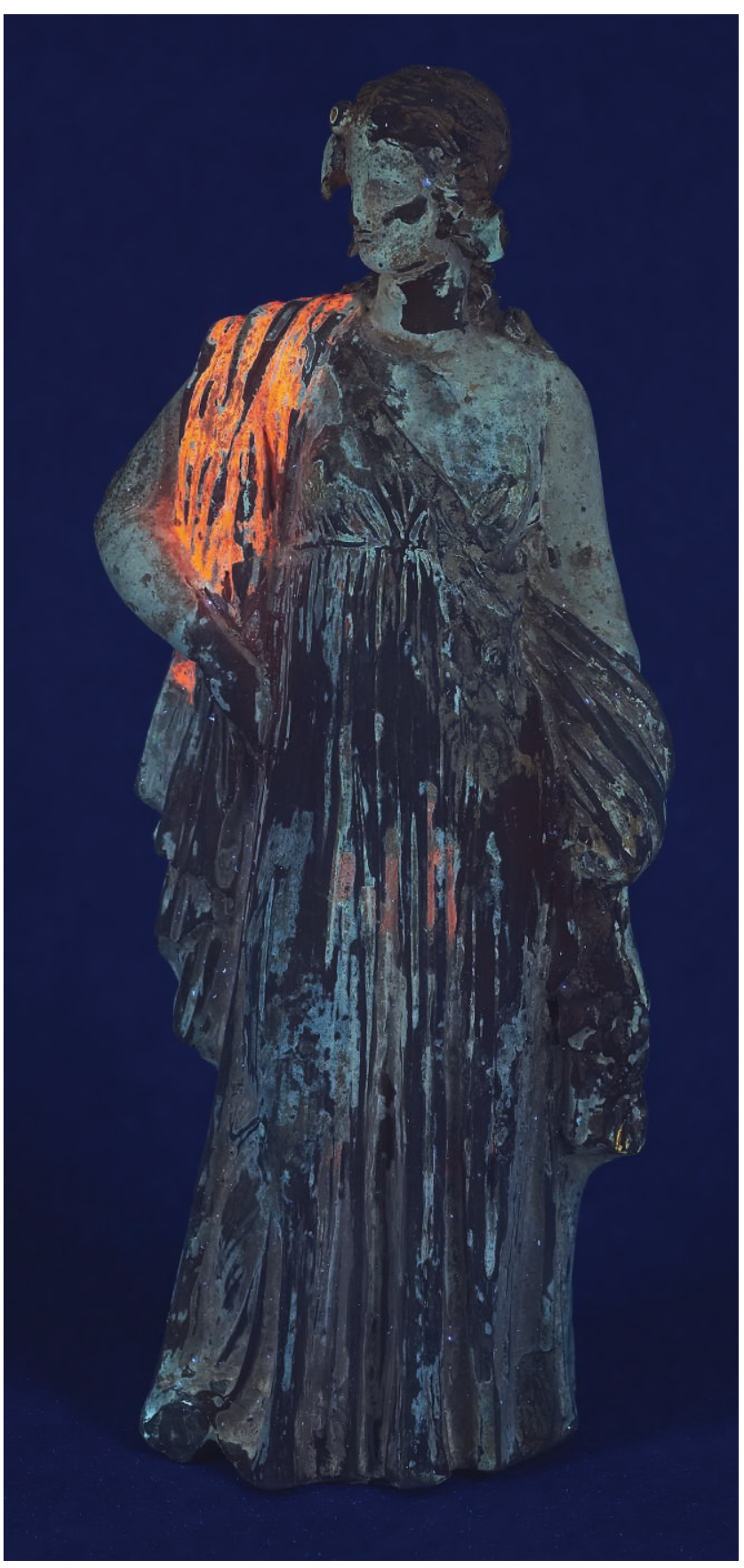

Fig. 1 b. Idem sous UV.

(c) C2RMF/Anne Chauvet.

demeurée quelque peu en friche ${ }^{5}$. On devine pourtant combien il importe de distinguer le travail de création de remaniements ultérieurs, sur des artefacts que l'archéologie et la muséologie modernes qualifient «d'originaux » et s'emploient à conserver comme tels. Et ne convient-il pas aussi d'intégrer ce nouvel angle d'approche dans les réflexions sur la fonction des objets? Tel a été notre objectif en développant les recherches sur des cas de repeints antiques, repérés au Louvre dans le cadre de la préparation à l'exposition Tanagra. Mythe et archéologie (Louvre, 2003), notamment sur la statuette de 
Ménade dite Dame Baillehache (fig. 1, a et b). La collection du Louvre offre, comme on le sait, un terrain d'étude privilégié par le nombre et la variété des objets; elle se distingue aussi, notamment pour les séries issues des fouilles de Tanagra et de Myrina, par une certaine «virginité», selon le mot d'Olivier Rayet $^{6}$ : sans être totalement exempte de faux, de «jutages» et de repeints modernes (qui ont été d'ailleurs étudiés), elle a moins souffert de ces interférences que d'autres collections formées plus tard ${ }^{7}$.

\section{Questions de méthodologie}

\section{L'examen en microscopie vidéo numérique}

Ce dont A. Laumonier rêvait, à savoir l'observation la plus attentive et l'illustration la plus exacte possible, garante d'un «travail scientifique précis ${ }^{8}$ », la technologie moderne le met désormais à notre disposition, avec l'emploi de microscopes vidéo numériques puissants, opérant en mode 2D et 3D. De la sorte, il devient possible d'étudier les strates sous différents angles et de mesurer leur épaisseur.

Si certaines données telles que la composition et le mode précis d'application des couches de surface ne peuvent être connues que par l'analyse d'échantillons préparés en coupes stratigraphiques, indispensables en pareil cas, la première étape n'en demeure pas moins l'examen le plus approfondi possible. C'est celle-ci dont nous rendons compte à présent, sur la base d'observations effectuées récemment (2013-2014), que des analyses compléteront ${ }^{9}$. L'histoire matérielle de l'objet doit être appréhendée dans sa globalité, en évaluant l'impact de l'enfouissement comme la part, souvent non documentée, des retouches modernes, elles-mêmes vieilles parfois de plus d'un siècle. La présence des sédiments archéologiques ainsi que l'observation des altérations dues à l'enfouissement sont évidemment essentielles pour attester l'ancienneté des couches observées. En règle générale, il convient de raisonner sur l'ensemble de l'œuvre, de manière à juger du caractère répétitif et cohérent ou non des observations. Les inconnues sont nombreuses, en effet, et bien des facteurs ont pu jouer, entre les aléas de la conservation au cours du temps et des irrégularités de stratigraphie dues à la manière dont l'artisan antique a procédé (en amincissant une couche par exemple jusqu'à la faire «mourir» de manière imperceptible).

Quelques critères techniques peuvent aider à mieux différencier la mise en couleur initiale de reprises diachroniques.

\section{Mise en couleur initiale: préparation, juxtaposition et superposition de couches}

Aux époques classique et hellénistique, la pratique grecque habituelle était la suivante. Une fois opérée la cuisson, la surface de la figurine était enduite d'une couche de préparation, généralement blanche, sauf cas exceptionnels de préparation grise comme à Myrina ${ }^{10}$. Puis elle était peinte, d'ordinaire par juxtaposition d'aplats de couleur unie, posés en couches très fines sur les meilleures pièces afin de ne pas empâter le modelé et de conserver la luminosité du coloris. L'application de feuilles d'or (ou d'autres feuilles métalliques telles que l'étain), en rehauts ou en traitement de surface intégral, s'opérait également à ce stade ${ }^{11}$. La nature du (ou des) liant employé, vraisemblablement soluble à l'eau (gomme végétale, colle animale, œuf, miel?) échappe encore malheureusement souvent aux tentatives de caractérisation par analyse. La qualité du travail plastique et de la mise en couleur était évidemment variable, du coloriage sommaire à la «kosmèsis» (embellissement, parure) la plus raffinée, mettant en jeu une réelle maîtrise technique.

Quoique moins fréquente, la pratique de superposer des couches colorées n'était pas exclue. Sur les pièces soignées des ateliers d'Attique, de Béotie ou de Myrina, pour ne prendre que ces exemples, il arrive que des motifs aient été peints les uns sur les autres, dans des tonalités différentes, pour restituer la richesse ornementale des vêtements ${ }^{12}$. Plus intéressant encore, le procédé consistant à monter une couleur en plusieurs couches a été observé sur certaines figurines. La Dame en bleu, pièce exceptionnelle de Tanagra des années 330-300 (inv. MNB 907), est ainsi drapée dans un manteau peint en bleu, ourlé d'une large bande dorée. Le bleu (bleu égyptien) a été appliqué sur un fond gris (mélange de kaolinite et de noir de charbon) lui-même posé sur la préparation blanche en kaolinite ${ }^{13}$. Comme des travaux récents l'ont souligné, l'emploi d'un tel fond coloré s'explique par le désir d'économiser certains pigments plus onéreux, tout comme par la recherche d'effets chromatiques. L'étude approfondie d'H. Brecoulaki sur la peinture grecque des tombes de Macédoine aux IV ${ }^{\mathrm{e}}$ et III ${ }^{\mathrm{e}}$ siècles av. J.-C. a parfaitement éclairé cette science de la superposition des couleurs que possédaient les peintres grecs et que les textes antiques désignaient par le terme d'«épivolè ${ }^{14} »$.

C'est, comme on l'a dit plus haut, la recherche d'intensité et d'éclat qui explique aussi l'association d'une peinture rose sur rouge. Le rose vif, l'un des tons les plus privilégiés à l'époque hellénistique, correspond en général à la garance (caractérisée par sa vive fluorescence orangée sous UV) ; posé sur un fond d'un rouge puissant, il produisait un coloris vibrant. Ce procédé, attesté par ailleurs dans la grande peinture, était visiblement en faveur dans les ateliers de Délos et de Myrina à l'époque hellénistique tardive. Regardons par exemple, au musée de Délos, une grande statuette de Tychè (inv. A 312). Elle porte une tunique longue (chiton) blanche, serrée par une ceinture sous les seins et ornée de bandes peintes en rose de garance sur un fond rouge à base de fer; appliqués sur le rose en dernier lieu, de légers traits rouges soulignent l'animation des plis (fig. 2) ${ }^{15}$. À Myrina, la "phainoméride », l'une des plus belles statuettes issues du Tombeau B (Louvre, Myr 163) témoigne de ce même procédé chromatique (rose sur rouge), ainsi que, dans les motifs de bandes qui ornent le bas de la tunique, d'un jeu de superpositions particulièrement savant ${ }^{16}$. 


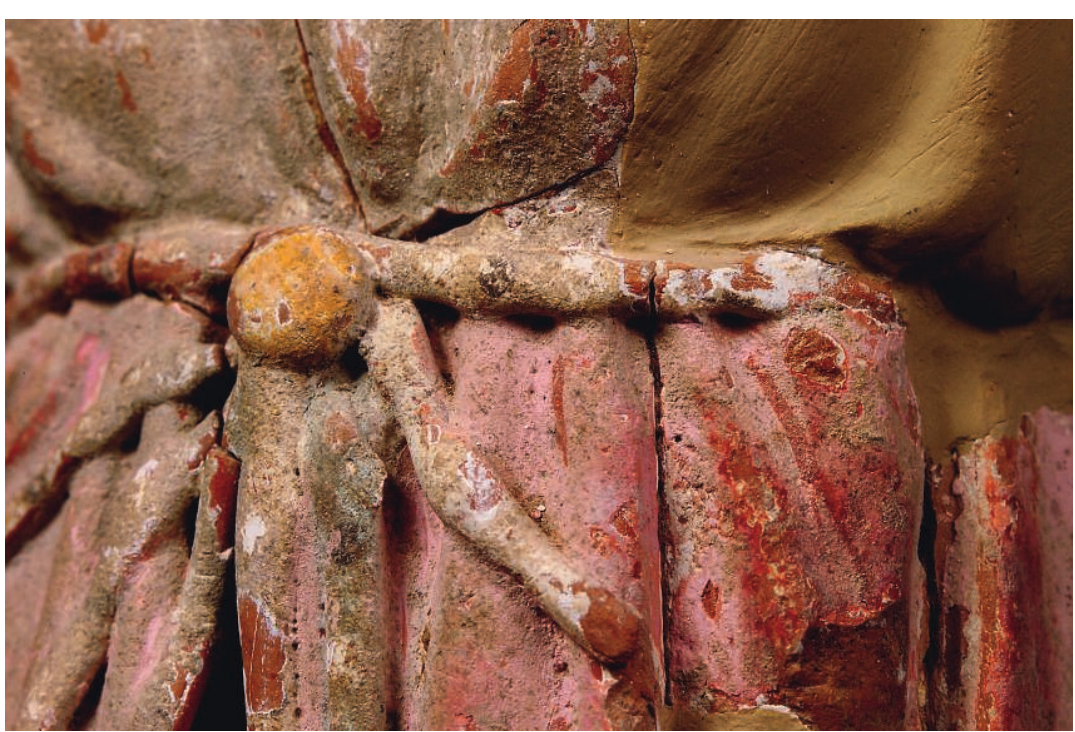

Fig. 2. Figurine de

Tychè, Délos, $\mathrm{II}^{\mathrm{e}} \mathrm{-}^{\mathrm{er}}$

siècles av. J.-C., terre

cuite peinte, Musée de

Délos, A 312. Détail de

la tunique sous la

ceinture: bande peinte

en rose de garance sur

un fond rouge, le tout

sur préparation

blanche. Ajoutés en

touches finales sur le

rose, des traits de

peinture rouge

animent les plis du

vêtement.

(c) EFA/Philippe Collet.

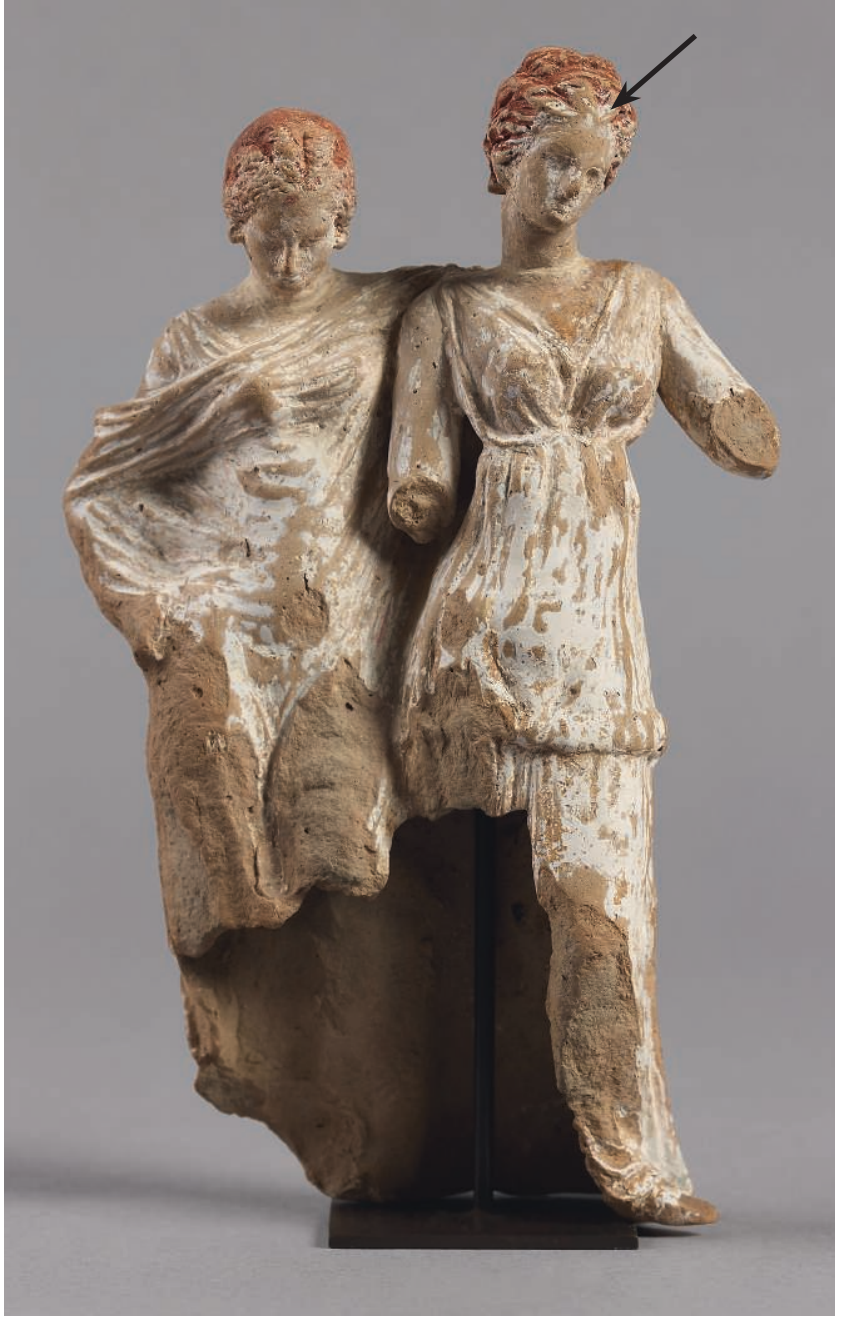

Fig. 3 a. Groupe de femmes (Déméter et Coré?), Attique (?), $\mathrm{III}^{\mathrm{e}}$ siècle av. J.-C., trouvée à Smyrne, terre cuite peinte, H. $19 \mathrm{~cm}$, Louvre, CA 774. Vue d'ensemble. La flèche indique l'emplacement de la fig. 3b. () Musée du Louvre, Dist. RMN-Grand Palais/Anne Chauvet.

\section{Reprises diachroniques: superpositions liées à des repeints}

Un critère permet d'affirmer la volonté de repeindre: c'est la présence, entre les états colorés, d'une nouvelle couche de préparation opaque, masquant et effaçant de la vue la polychromie antérieure. L'appui du microscope s'avère ici précieux lorsque les couches sont très fines et que le repeint s'est effectué à l'identique, sans changement de tonalité qui attire l'attention: c'est le cas pour un groupe de femmes enlacées (Louvre, CA 774), où deux applications de brun-rouge sur préparation blanche ont été détectées sur la chevelure (fig. 3, a et b). Sur ce même groupe, l'hypothèse d'une modification des vêtements, passant de la peinture à la dorure, avait été précédemment avancée sur la base d'un examen binoculaire opéré à plus faible grossissement ${ }^{17}$. Comme la trouvaille provient de

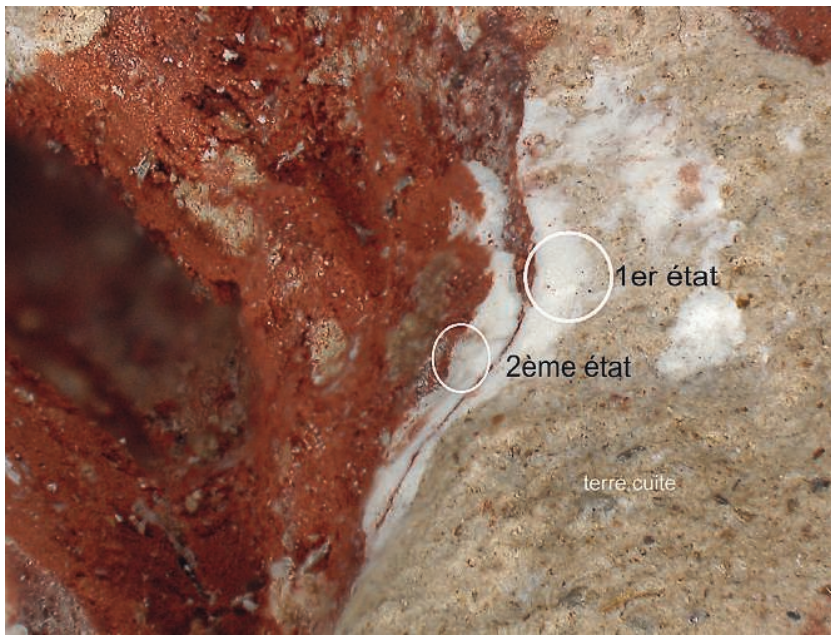

Fig. 3 b. Sur le groupe CA 774, repeint antique de la chevelure (microscope numérique 3D, x 100). Du support à la couche de surface: terre cuite; premier état (peinture brun-rouge sur préparation blanche); deuxième état (peinture brun-rouge plus vif sur préparation blanche). ( C C2RMF/Brigitte Bourgeois. 
Smyrne, mais que les analyses d'argile indiquent vraisemblablement une production attique, il avait été proposé que la pièce, une fois importée, ait été mise au goût de la clientèle locale; on sait en effet combien les ateliers smyrniotes s'étaient spécialisés dans la production de figurines en terre cuite dorée ${ }^{18}$. L'approfondissement de l'étude récemment menée avec un microscope plus performant oblige cependant à renoncer à cette hypothèse tentante. Le groupe a bien fait l'objet d'une nouvelle kosmèsis dans l'Antiquité, mais le médium est resté la peinture. Ce qui avait été pris pour d'infimes restes de dorure ne correspond qu'à des brillances parasites, dues à des particules minérales présentes dans les concrétions d'enfouissement.

Dans bien des cas cependant, la complexité des phénomènes observés peut être grande, comme nous allons le voir maintenant.

\section{La Dame Baillehache (Louvre, inv. CA 2552)}

Au sein de la collection du Louvre, reprenons tout d'abord le dossier de la figurine dite Dame Baillehache, une Ménade, identifiée par la couronne de feuilles de lierre et la nébride (peau de faon) qu'elle portait (fig. 4, a et b). Elle est vêtue d'un chiton long et d'une écharpe (chlaina). Cette œuvre représentative du style dit «tanagréen" a été récemment attribuée, sur la base d'analyses physico-chimiques de l'argile, à un atelier attique et datée entre 330 et 200 av. J.-C. ${ }^{19}$

\section{État antérieur de la question}

Nous ne savons rien du lieu précis de découverte de la «plus belle Tanagra» de la collection d'Alfred Baillehache, qu'il légua au musée du Louvre en $1923^{20}$. À une date indéterminée, la figurine a été moulée et nettoyée; ces interventions, qui n'ont pas été notifiées dans les archives, ont malheureusement provoqué des arrachements de la couche superficielle $^{21}$.

Repérés au moment de la restauration par M.-E. Meyohas en 2002, les repeints antiques sont bien certifiés par la présence de couches de préparation blanche séparant les strates colorées. Une restitution de trois états successifs avait à l'époque pu être proposée ${ }^{22}$. Le premier état, le plus ancien, montrait un chiton bleu dont les plis sur le devant du corps, de la ceinture aux genoux, auraient été soulignés de jaune, avec une écharpe rouge-orangé. Le deuxième état aurait juste vu la modification de l'écharpe en rose pâle. Le troisième état, correspondant à la surface actuelle, aurait quant à lui fait l'objet d'une modification totale. Le chiton, désormais ceinturé par une cordelette rouge, serait alors devenu gris et l'écharpe rose vif (garance), tandis que la feuille d'or venait mettre en valeur les accessoires, déjà présents comme les baies de la couronne, ou nouveaux comme les boucles d'oreilles, le collier, le bracelet sur le bras gauche et les motifs de forme

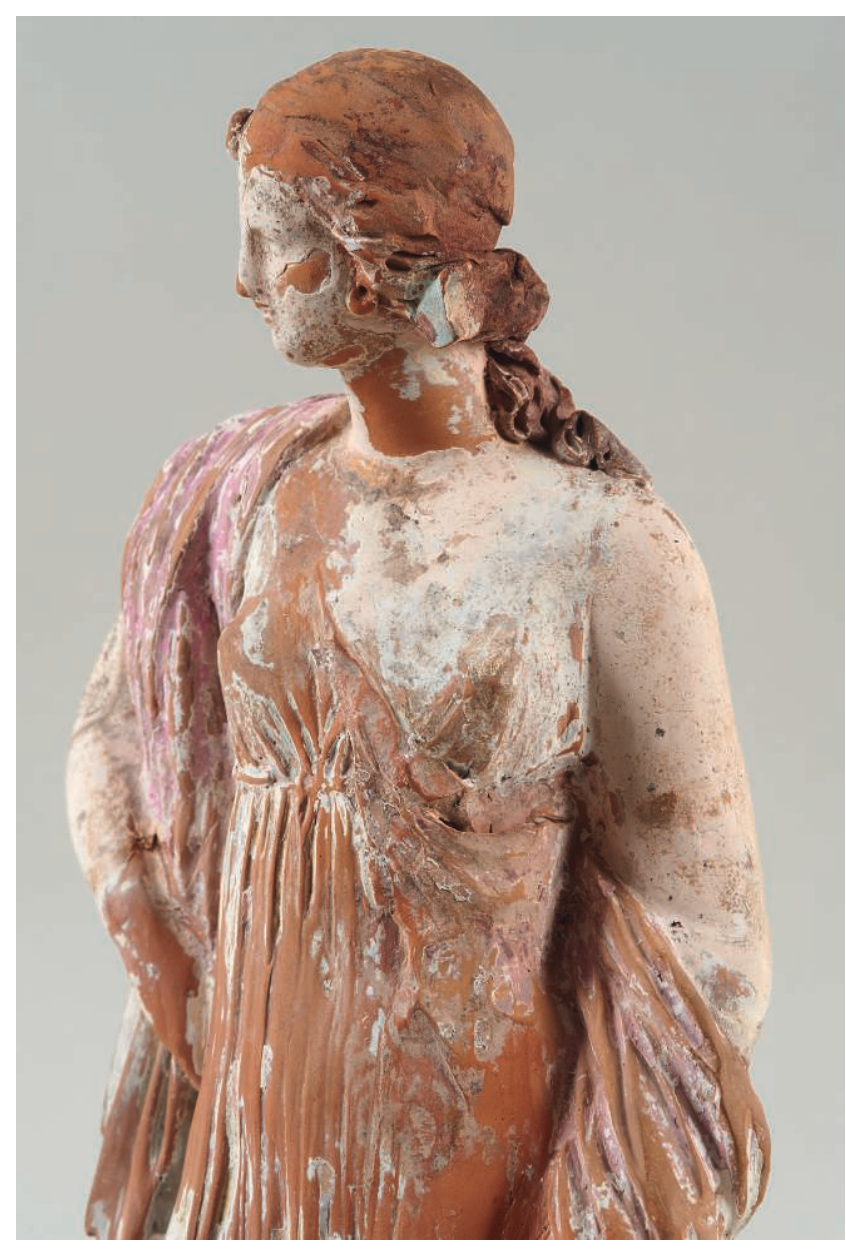

Fig. 4 a. Dame Baillehache, le buste et, sous le sein gauche, la zone de la nébride: la surface irrégulière de la terre cuite porte encore trace de la peinture qui a été appliquée après la chute de l'élément plastique originellement rapporté. (c) C2RMF/Anne Chauvet.

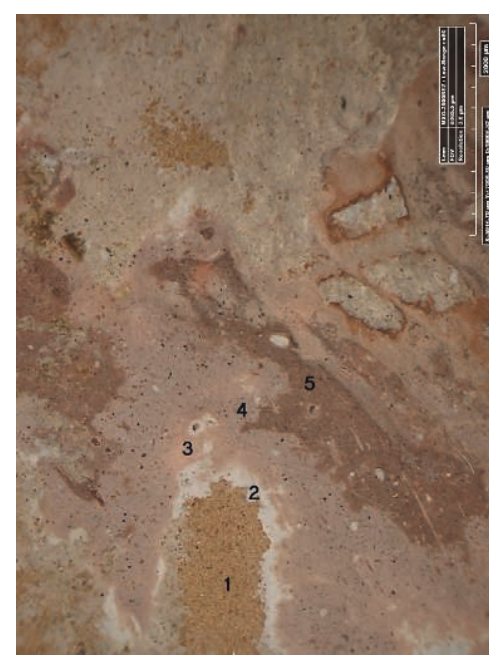

Fig. 4 b. Le pelage de la nébride peinte sur la zone accidentée (microscope numérique 3D, x 50). Du support à la couche de surface, couche 1: terre cuite, 2: préparation blanche, 3 : couche picturale rosâtre, 4 : couche picturale gris rosé, 5 : motifs du pelage en brun. (c) C2RMF/Brigitte Bourgeois. 
maintenant indéfinissable sur la poitrine. On avait alors suggéré que la nébride, originellement modelée à part et rapportée, se soit détachée durant la cuisson, obligeant ainsi l'artisan à rhabiller la figure par la peinture; le sein gauche aurait alors été tout de suite couvert par une couche de bleu puis, ultérieurement, par du gris.

\section{Nouvelles données}

La reprise de l'examen avec le microscope 3D KH 8700 en 2014 , tout en confortant certaines observations, nous a permis d'affiner la réflexion, mais aussi de poser de nouvelles interrogations.

Constatons tout d'abord une certaine incohérence entre le nombre d'états observés selon les zones, sur la face et le revers. La reprise, par deux fois, de l'écharpe, bien identifiée au revers de l'épaule droite (fig. 5, a et b), a aussi provoqué, sans doute lors du dernier état, la réfection de la mèche de cheveux qui tombe sur l'épaule, comme le prouvent les deux états de brun-rouge sur préparation blanche. On peine cependant à clairement identifier ces trois étapes sur l'avers où seuls l'état ancien, rouge, et le dernier état, rose vif, sont bien visibles. Dans la retombée de l'écharpe, sous le poignet droit, on observe également trois couches, semble-t-il, passant d'un bleu à deux gris (fig. 6) -mais d'autres zones du chiton, sur la face, n'attestent qu'une seule reprise. Concernant les carnations, deux états sont bien visibles sur le visage, mais certains clichés pris au revers de l'épaule gauche ne suggèreraient-ils pas plutôt trois états (fig. 7) ? Enfin, l'examen a mis en évidence une autre couche intermédiaire de couleur beige, non repérée jusque-là : elle est ponctuellement localisée au revers de l'écharpe entre la couche rose pâle (deuxième état) et la couche rose vif (troisième état) (fig. 5 b et 6). C'est elle qu'on retrouve aussi en partie basse de l'écharpe, toujours au revers, sur la première couche grise recouvrant l'état initial bleu pâle du chiton.
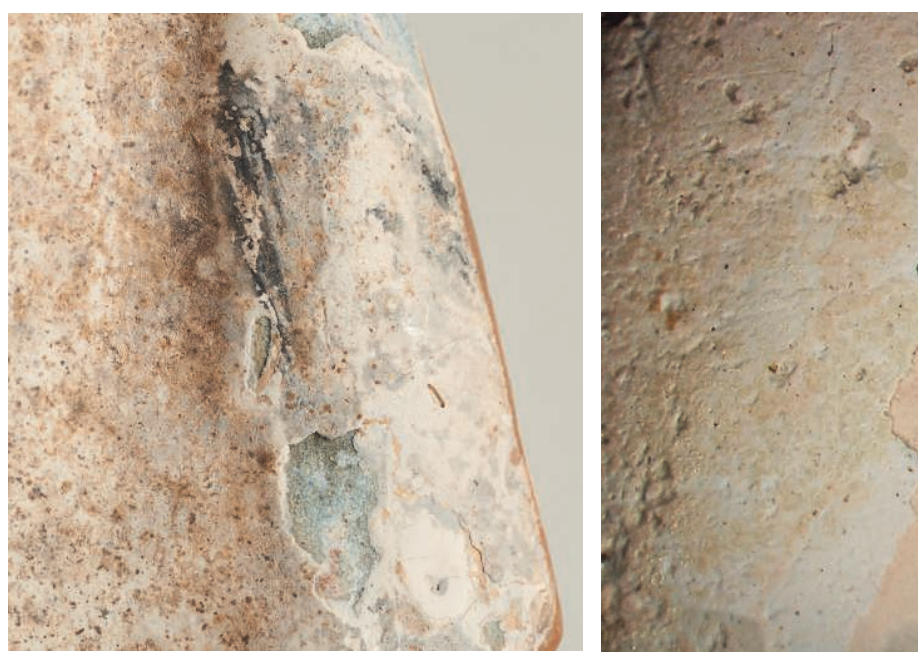

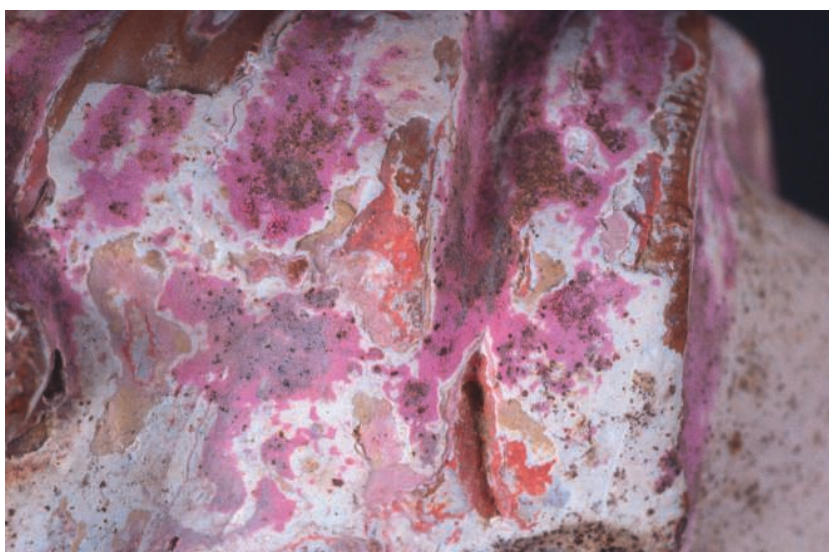

Fig. 5 a. Dame Baillehache, revers de l'écharpe sur l'épaule droite, montrant les trois états antiques de polychromie. (C) C2RMF/Anne Chauvet.

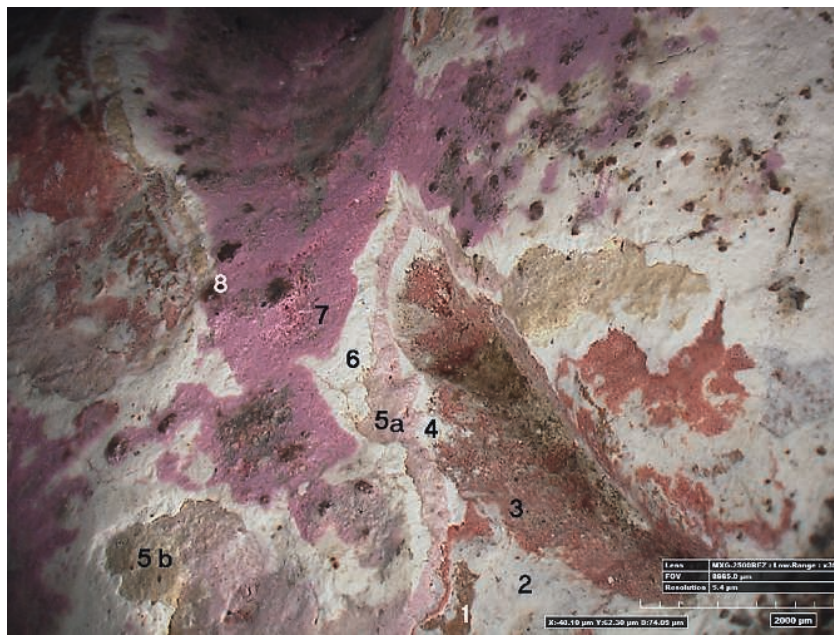

Fig. 5 b. Repeints de l'écharpe (microscope numérique 3D, x 35). Du support à la couche de surface, couche 1 : terre cuite,

2-3: premier état (couche picturale rouge sur préparation blanche), 4-5: deuxième état (couche picturale rose pâle (5 a) et beige (5 b) sur préparation blanche), 6-7: troisième état (couche picturale en rose de garance sur préparation blanche), 8: dépôts noirs d'enfouissement. (c) C2RMF/Brigitte Bourgeois.

Fig. 6. Dame Baillehache, repeints gris sur le premier
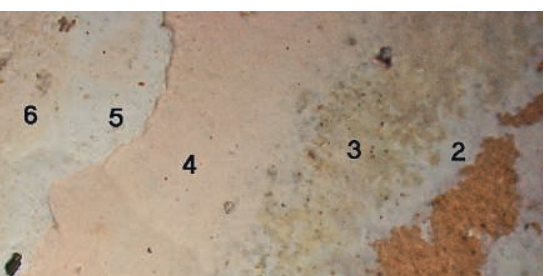

.

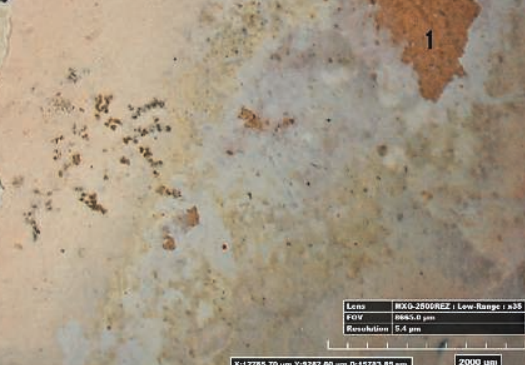

Fig. 7. Dame

Baillehache, repeints des carnations (microscope numérique 3D, x 35). Du support à la couche de surface, couche 1 : terre cuite, 2-3: premier état (couche picturale ivoire sur préparation blanche), 4: deuxième état (?) (couche picturale rosée), 5-6: dernier état (couche picturale ivoire rosé sur préparation blanche). (C) C2RMF/ Brigitte Bourgeois.

état bleu de la tunique, au revers, sous la main droite. (c) C2RMF/Anne Chauvet. 


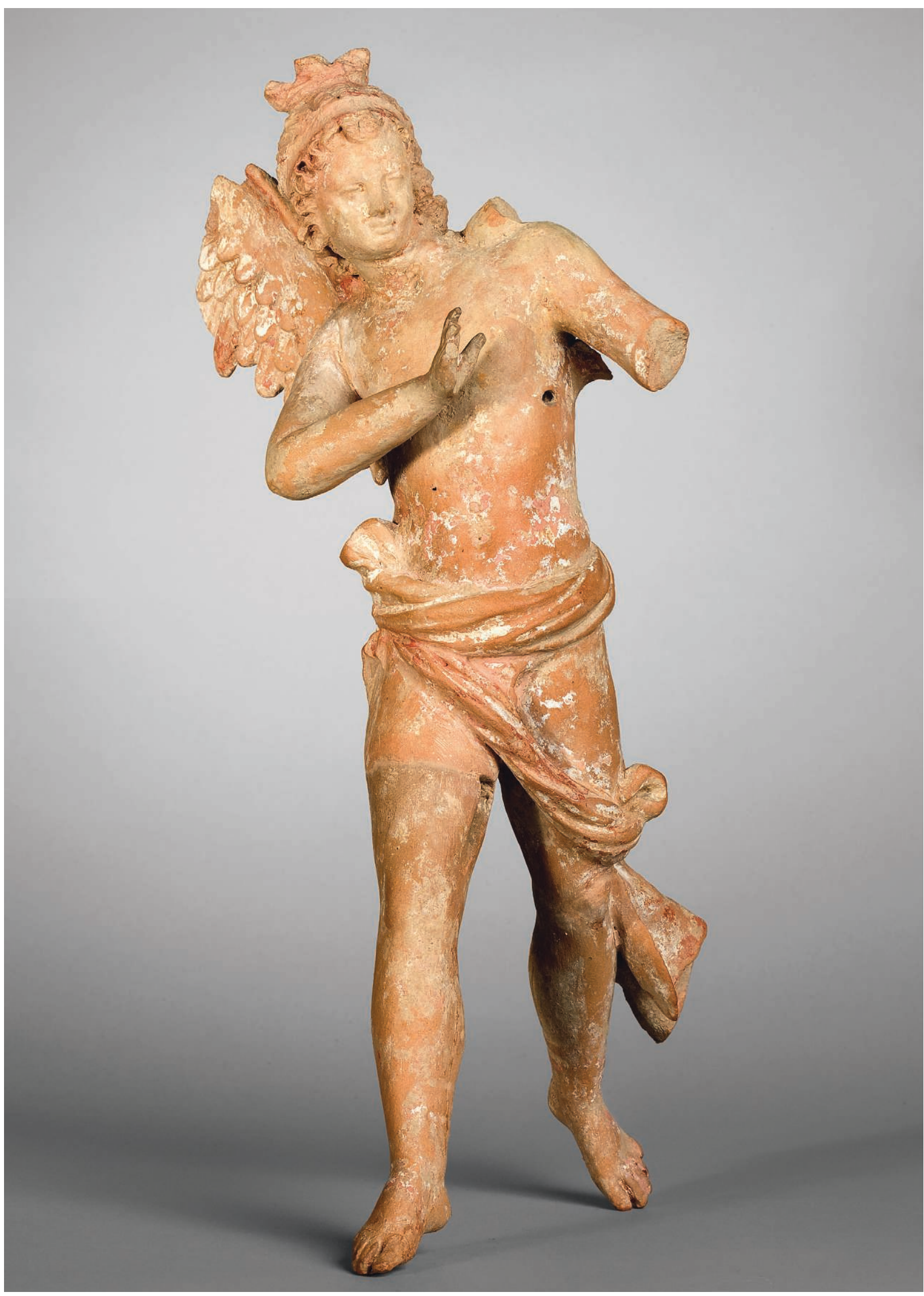

Fig. 8. Figurine d'Éros volant, Priène, vers 150 av. J.-C., terre cuite peinte et dorée, H. $28,3 \mathrm{~cm}$. Louvre, CA 790 bis. Vue d'ensemble. ๑ Musée du Louvre, Dist. RMN-Grand Palais/Daniel Lebée/Carine Déambrosis. 
Ces disparités entre les différentes zones posent question: avons-nous affaire à des repeints partiels ? Le premier décor s'était-il écaillé par endroits ou bien, autre hypothèse, l'artisan a-t-il choisi d'éliminer partiellement l'état antérieur par un grattage plus poussé sur la face, puisque celle-ci était évidemment plus visible?

Si certaines interrogations persistent, il n'en demeure pas moins qu'un certain nombre de données nouvelles ont pu être collectées; elles confirment pleinement l'attestation de jeux picturaux sur cette œuvre, ainsi que le rôle joué par la polychromie dans la «réparation » de l'accident marqué par la chute de la nébride.

À examiner le grand pli jaune central du chiton, posé sur la préparation sans trace apparente de bleu sous-jacent, il est clair en effet que les couleurs ont été réfléchies et posées de manière différenciée selon les zones: quelques plis ont ainsi été volontairement laissés en réserve au moment de la première mise en couleur (bleue) du chiton afin de privilégier certains effets lumineux. Et la présence ponctuelle de traits rouges sur ces mêmes plis relèverait du même procédé. Les rehauts beiges rosés (dont la garance est repérable grâce à sa fluorescence, voir fig. 1 b) présents dans les plis au niveau médian du chiton permettaient là aussi ces touches de lumière.

La récente étude a, d'autre part, permis de mieux comprendre la reprise de la zone irrégulière, sous le sein gauche, où une nébride avait été rapportée à l'origine (fig. 4, a et b). En effet, sur la zone accidentée, a été appliquée, par-dessus une fine préparation, une peinture gris rosâtre (montée en deux couches) couverte ici et là de taches de couleur brune évoquant le pelage d'une peau de bête. Cette même reprise pourrait avoir aussi vu la pose d'une très fine couche de peinture grise, aujourd'hui à peine sensible, sur la carnation du sein gauche, sous lequel nul grain de bleu égyptien n'est perceptible; c'est la preuve que le sein gauche était bien dénudé lors du premier état présentant un chiton bleu. Au moment de l'accident ayant provoqué par la suite la chute de la nébride, le haut du sein a donc été «rhabillé» par la peinture grise, et la zone d'arrachement de l'argile a servi à évoquer le relief de la peau de bête, elle-même rendue par une teinte rosâtre et ponctuée de taches ocre-brun. Nul souci de pudeur ici ni modification d'identité de la figure qui se définit toujours, par sa couronne de lierre l'associant à Dionysos et par sa dépouille animale, comme une ménade; l'habillage du sein est plutôt à lire comme une volonté esthétique sans que l'on puisse en donner les réelles motivations.

Il paraît enfin clair, à voir la présence de la laque de garance sur le seul côté droit de l'écharpe (marquée sur le cliché UV (fig. 1 b) par la fluorescence orange), alors que le pan enroulé autour du poignet gauche en est dépourvu, que l'artisan a délibérément repris la pièce afin de donner un coup d'éclat sur ce côté, en jouant ainsi de l'attitude générale de la figurine, la tête nettement tournée vers la droite et la pose hanchée par le contrapposto. Le regard sollicité par cet éclat oubliait ainsi quelque peu l'accident de la nébride dont la reprise par la peinture ne pouvait être qu'imparfaite.

\section{L'Éros de Priène (Louvre, CA 790 bis)}

Le second dossier concerne une statuette d'Éros (fig. 8) récemment présentée dans le cadre de l'exposition D'Izmir à Smyrne $e^{23}$. Entrée au Louvre en 1896, elle a été rattachée à la production de Priène (Turquie) et est datée des années 150-135 av. J.-C. La figuration du dieu adolescent s'inspire du type statuaire de l'Éros à l'arc attribué au sculpteur Lysippe (original à situer entre 338 et 335 av. J.-C.). Si les traces de peinture et de dorure que conserve la figurine avaient été repérées de longue date, ce n'est que lors de la préparation de l'exposition de 2009 que la restauratrice, Marie-Emmanuelle Meyohas, a noté la présence de repeints dont Isabelle Hasselin-Rous a rendu compte dans le catalogue ${ }^{24}$.

La reprise des carnations, peintes en jaune-brun dans le premier état, en rose dans le second, apparaît à dire vrai de manière évidente - et cohérente - sur le corps du dieu adolescent. Il suffit d'un examen à l'œeil nu pour s'en convaincre, en particulier sur le torse, dans la partie gauche de l'abdomen (fig. 9 a). Le recours au microscope s'avère néanmoins utile pour évaluer l'épaisseur des couches et visualiser le mélange de grains blancs, rouges, roses formant la peinture du second

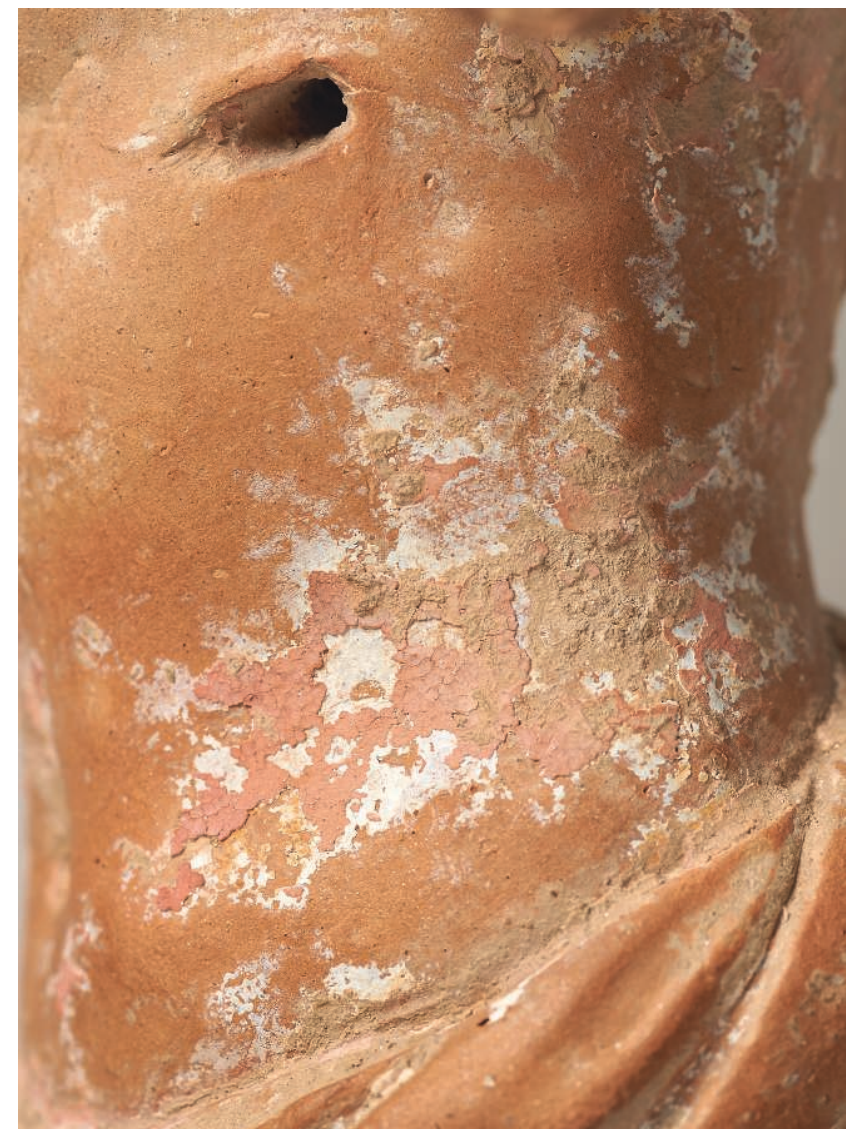

Fig. 9 a. Éros de Priène, partie gauche du ventre, repeint antique des carnations. (c) C2RMF/Anne Chauvet. 


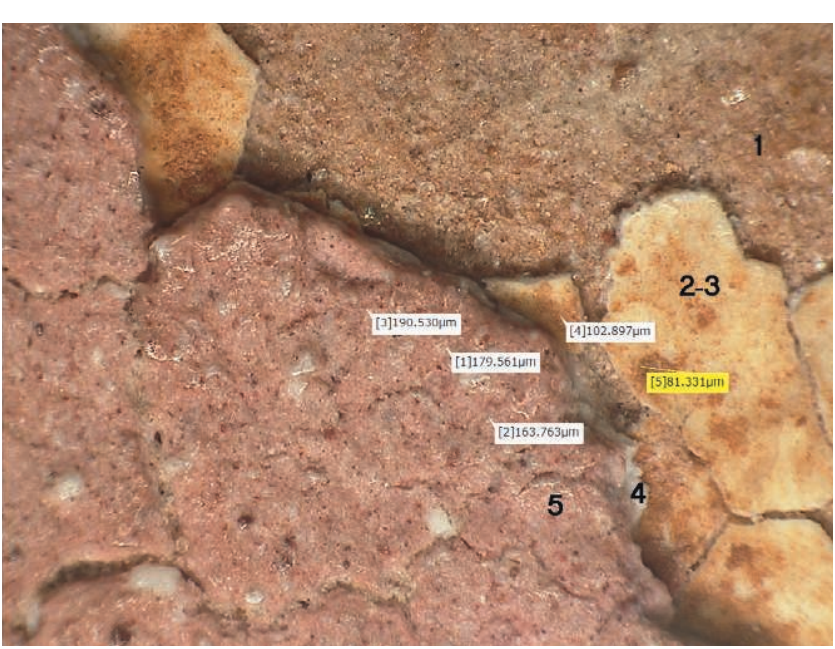

Fig. 9 b. Idem, vue au

microscope numérique 3D

(x 100). Du support à la

couche de surface, couche

$1:$ terre cuite, $2-3$ : premier

état (couche picturale

jaune-brun sur

préparation blanche),

4-5: second état (couche

picturale rose

sur préparation blanche).

L'épaisseur du repeint

avoisine les 80 microns.

(c) C2RMF/Brigitte

Bourgeois.

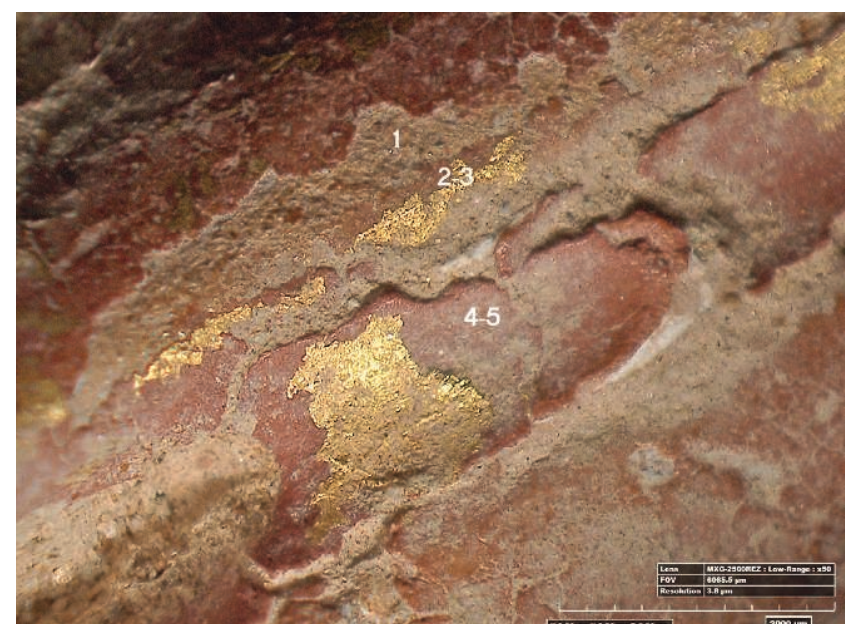

Fig. 11. Éros de Priène, pan de draperie, re-dorure ponctuelle (?) (microscope numérique 3D, x 50). Du support à la couche de surface, couche 1 : terre cuite,

2-3: premier état (feuille d'or sur assiette rouge), 4-5: second état hypothétique (nouvelle application de feuille d'or sur assiette rouge sur préparation blanche).

(c) C2RMF/Brigitte Bourgeois.

Qu'en était-il du drapé, de ce manteau enroulé autour des hanches dont un pan glisse en travers des cuisses pour flotter librement contre le genou gauche? Si des restes de dorure sont bien repérables là aussi à l'œeil nu, il n'en faut pas moins l'aide du microscope pour mieux comprendre la stratigraphie. L'hypothèse d'un repeint rouge sur la dorure, évoquée dans le catalogue de 2009, doit être écartée. L'examen montre en général que la feuille d'or a été appliquée sur une assiette rouge plus ou moins épaisse qui repose ou non, selon les zones, sur une préparation blanche. Si re-dorure il y eut, elle n’a été que ponctuelle. Encore reste-t-il délicat d'interpréter certaines images. Qu'on regarde par exemple le pli du manteau passant sous le sexe (fig. 11). Des vestiges d'une feuille d'or y subsistent, collés sur une mince assiette rouge qui repose directement sur la terre cuite; sur cette première dorure, apparemment usée, il semble qu'ait été réappliquée une nouvelle assiette rouge, épaisse et craquelée, sur préparation blanche. 


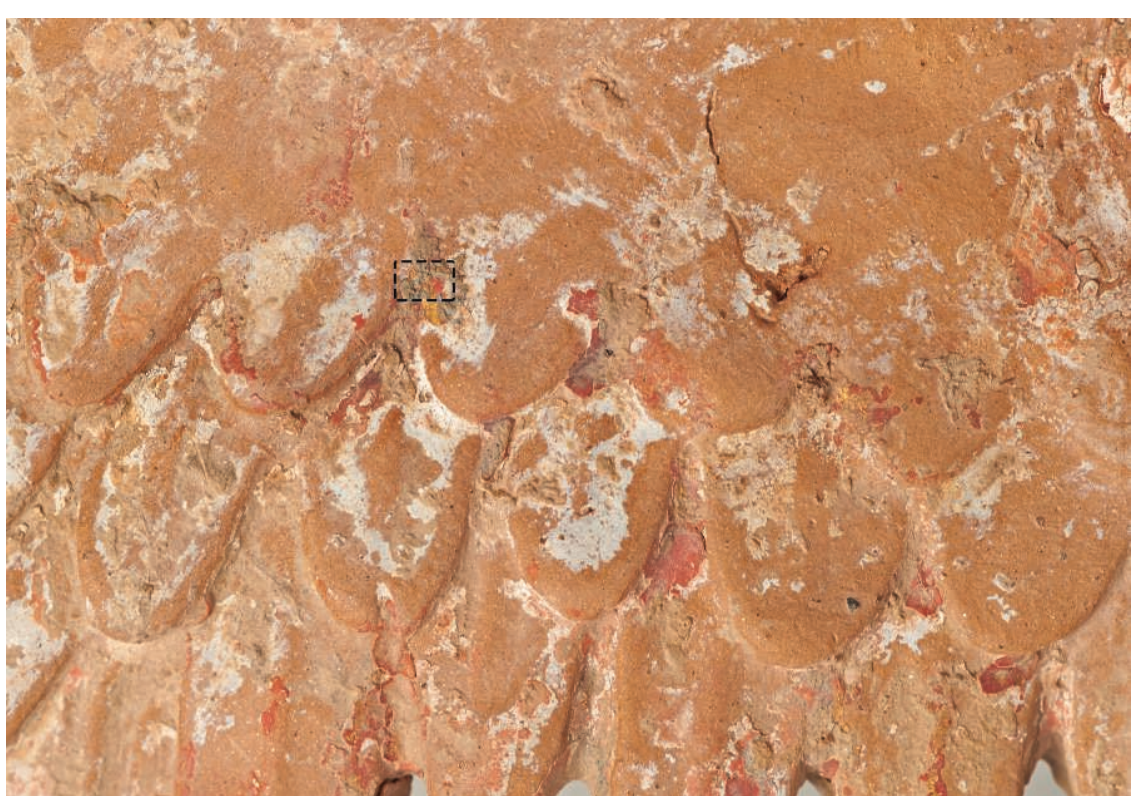

Fig. 12 a. Éros de Priène,

détail de l'aile droite.

(c) C2RMF/Anne Chauvet.
L'observation de l'aile droite, la seule conservée (fig. 12 a), conforte elle aussi l'hypothèse d'une reprise de polychromie - même si les traces demeurent trop ténues, malheureusement, pour permettre de restituer avec certitude l'état originel. Ce qui importe surtout, c'est de noter comment, dans le second état, l'artisan a associé peinture et dorure pour accentuer vraisemblablement des effets de clair-obscur. Des dégradés de tons orangés, rouges et bruns, qu'on dirait peints à l'ocre ${ }^{25}$, sont encore visibles par endroits; une trace nette subsiste notamment près de l'attache de l'aile et de l'épaule droite. Sur les deux rangées de petites plumes, des touches de bleu (bleu égyptien) ont été appliquées, scandant chacune des dépressions; tantôt elles sont encore visibles à l'œil nu, tantôt elles ne sont plus discernables qu'au microscope. De plus, la matière picturale bleue apparaît fréquemment associée à des restes de dorure - le bleu étant bien posé sur la feuille d'or (fig. 12 b). La prospection microscopique (à grossissement x 50-100) n'ayant montré aucune trace de bleu en dehors de ces zones bien localisées, on peut en conclure que l'aile n'a pas été repeinte en bleu sur toute son étendue. Il faut envisager plutôt un procédé pictural visant à animer d'ombres bleutées une aile peinte et dorée tout à la fois. L'examen mené en son temps par Dorothy Burr sur des pièces de Myrina, que nous rappelions en début d'article, n'avait-il pas déjà relevé des indices de jeux picturaux sur des ailes de Victoires?

\section{Conclusion: examen de la matière et tentatives de «recontextualisation»}

L'examen attentif de ces figurines par les méthodes avancées qui s'offrent désormais à nous est donc riche d'enseignements; il nous permet aussi de concevoir la complexité de leur histoire. Pour la Baillehache, les repeints, comme leur localisation, sont donc assurés pour le premier et le dernier état dont le revers donne une image sans doute plus complète. Pour autant,
Fig. 12 b. Éros de Priène, sur l'aile, touche de bleu mêlé de blanc sur une dorure à la feuille, appliquée sur une couche rouge sur préparation blanche (microscope numérique 3D, $\mathrm{x} 100)$. (C) C2RMF/Brigitte Bourgeois.

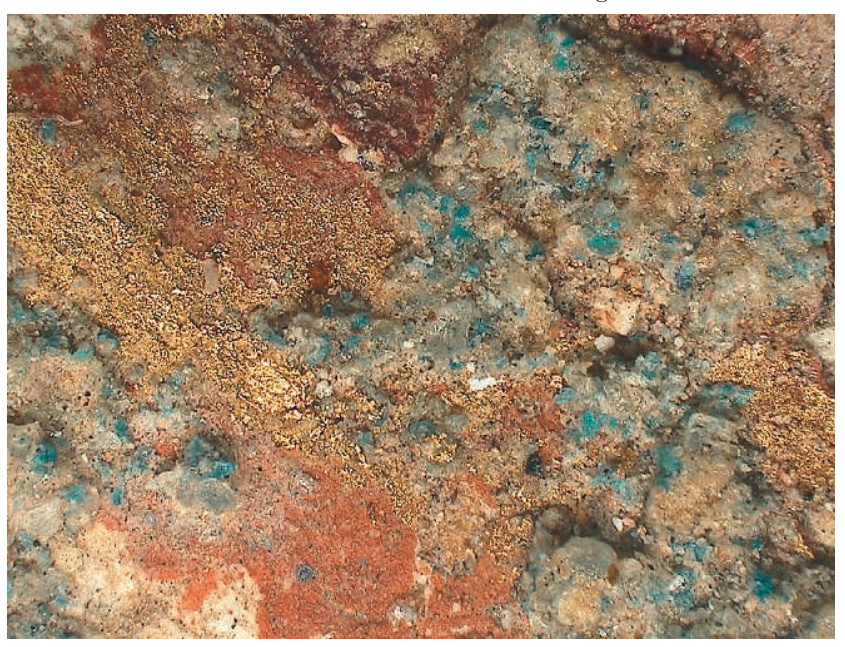

si l'on peut imaginer qu'une des réfections de cette œuvre a été provoquée par un accident (chute de la nébride), pour elle comme pour l'Éros de Priène, les raisons des autres réfections, leurs chronologies respectives, et, d'une manière générale le contexte de ces figurines comme leur fonction nous restent inconnus. Ces réfections pouvaient-elles par ailleurs suivre une mode vestimentaire ou des goûts propres à une époque: utilisation préférentielle du rose vif, désaffection (partielle) du bleu égyptien au profit d'autres teintes?

L'étude de la matière ouvre par ailleurs de nouveaux champs de réflexion. En effet, parallèlement à l'examen de la surface, l'enquête archéométrique nous a également permis ces dernières années d'isoler en Grèce continentale ou micrasiatique certains ateliers ${ }^{26}$. Ainsi l'origine attique de la Dame Baillehache confirmée par les analyses d'argile revêt-elle une importance considérable dans l'histoire du style dit «tanagréen ", dont cette figurine devient un fleuron. Quant à l'Éros du Louvre, 
sa similarité avec des figurines tirées de moules parallèles ou très proches à la provenance assurée (elles ont été trouvées à l'intérieur d'une pièce à vocation religieuse dans la maison 29 de Priène ${ }^{27}$ ), permet d'orienter notre réflexion et d'oser la confrontation de deux pièces pourtant chronologiquement et géographiquement bien éloignées. Car ces étonnantes (et rares?) reprises de polychromie, tout comme l'exceptionnel état de conservation des deux figurines ${ }^{28}$, pourraient permettre d'identifier ces statuettes comme les éléments d'un culte privé dans le cadre d'une maisonnée, pratique dont l'existence est bien connue. Si cette hypothèse n'est guère étonnante pour la pleine époque hellénistique, à laquelle appartient l'Éros, elle serait en revanche, pour la Baillehache, l'un des rares cas ainsi révélés pour la fin du $\mathrm{IV}^{\mathrm{e}}$ siècle ou le nécessairement une «durée de vie » de l'œuvre qu'il nous est impossible de quantifier et qui, parallèlement, suppose une forme de visibilité, soit dans un contexte domestique sacré, ainsi que nous venons de l'avancer, soit encore, et il ne faut pas totalement l'exclure du moins pour la Ménade dont le contexte reste obscur, dans l'atelier de l'artisan.

Ainsi l'étude de la couleur sur un matériel trop souvent négligé permet-elle d'ouvrir sur un certain nombre de problématiques qui dépassent largement la simple connaissance technique. Là où les textes se taisent, c'est donc paradoxalement le produit fini hors contexte qui pourrait aujourd'hui nous donner les meilleurs indices de certaines pratiques artisanales. L'étude approfondie de la matière devient la source d'un questionnement fécond qui nous mène des gestes des artisans jusqu'aux pratiques cultuelles et sociales de l'Antiquité. figurines en contexte domestique sont plus rares). Il est en tout cas clair que ces remises en couleurs impliquent

\section{Notes}

1. A. Rouveret et autres contributions dans Descamps-Lequime, 2007.

2. Burr, 1931, voir notamment p. 32 : superposition de rose et rouge, et repeint sur la Victoire cat. $\mathrm{n}^{\circ} 72$ (Boston, Museum of Fine Arts, inv. 01.7750) ; p. 34: ombres rouges («fine red brush strokes») sur les plis du chiton de la Victoire cat. $\mathrm{n}^{\circ} 70$.

3. Laumonier, 1956. Le corpus, comprenant environ 1400 numéros, provenait des fouilles menées dans l'île par l'École française d'Athènes depuis les années 1870.

4. Vingt-trois dossiers de figurines ont été inclus dans le programme de recherche, en raison des nombreuses affinités qu'elles présentent avec les marbres. Les objets ont été étudiés en vidéo-microscopie et soumis à quelques analyses. Certains cas de reprises antiques ont été illustrés dans des publications, voir notamment Bourgeois Jockey, 2000, 2007, Bourgeois, Jockey, Karydas, 2009, etc.

5. Ainsi dans les éditions successives du catalogue de l'exposition itinérante Bunte Götter. Outre les travaux actuels de Clarissa Blume (Blume, 2014), voir auparavant, sur la mise en couleur des terres cuites, Brinkmann cat. exp. 1996, et l'article fondateur de Higgins, 1970.

6. Rayet, 1878 .

7. Jeammet et Mathieux dans Jeammet (éd.), 2007, sur l'apparition des premières Tanagréennes; analyses de polychromie (antique et moderne) et TL dans Bouquillon et alii, 2003 et 2007; Bourgeois, 2007 pour les anciens traitements de restauration; et Zimmer, 1994, pour la collection de Berlin.

8. Laumonier, REG 1956, vol. 69, p. 207208, dans son compte rendu du tome I du catalogue des terres cuites du Louvre par S. Besques.

9. Étude réalisée avec un microscope Hirox KH-8700 (caméra numérique haute définition à 2,1 Mo, lentille zoom Revolver MXG-2500REZ 35x-2500x, capture d'image en mode multi-focus 3D). Tous nos remerciements à Dominique Robcis
(C2RMF, Département Restauration) pour son aide. De nouvelles analyses sont en cours sur les matériaux de la couleur (peinture, dorure des figurines en terre cuite grecques) dans le cadre du programme de recherche PilinA (LabEx Patrima) porté par S. PagèsCamagna et B. Bourgeois avec V. Jeammet et C. Fourdrin - post-doctorat.

10. Jeammet, Knecht, Pagès, 2007.

11. Bourgeois, Jeammet, Pagès, 20122013.

12. Voir par exemple une statuette béotienne de femme debout drapée (Louvre, MNB 447), Thèbes?, 330-200 av., dans Jeammet (éd.), 2003, n 127 p. 182 et Jeammet (dir.), 2010, n 205, p. 253.

13. Jeammet (dir.), 2010, n 91, p. 122 et p. 118 et Jeammet (éd.), 2003, n 132; analyses de S. Colinart, voir Bouquillon et alii dans Jeammet (éd.) 2003, p. 299-300. Rapport d'analyses de S. Colinart, C2RMF, dossier FZ 11454.

14. Brecoulaki, 2000 et 2006 . Rouveret, 2007.

15. Tychè A 312: Laumonier 1956, $n^{\circ}$ 301, pl. 31. Illustrée dans Bourgeois, Jockey, 2007, p. 185. Autres attestations à Délos sur le carquois d'un Apollon archer (inv. A 394), sur le tambourin d'une Cybèle trônante (inv. A 3305) etc.

16. Jeammet, Knecht, Pagès, 2007, p. 196-199 et Jeammet dans Østergaard, 2014 (éd.), p. 208-222.

17. Jeammet (dir.), $2010, \mathrm{n}^{\circ} 131$, p. 171 , d'après les observations faites par Ch. Devos.

18. Hasselin-Rous, Laugier, Martinez (dir.), 2009, et Bourgeois, Jeammet, PagèsCamagna, 2012-2013.

19. H. 27, $5 \mathrm{~cm}$, analyses A. Bouquillon, voir Jeammet (éd.), 2003, $\mathrm{n}^{\circ} 150$, et Jeammet (dir.), 2010, n² 207, Bourgeois dans Østergaard, 2014 (éd.), p. 190-207. C'est Athènes qui fut à l'origine de ce style ainsi dénommé en raison des découvertes à Tanagra (Béotie) de ce type de figurines au XIX ${ }^{\mathrm{e}}$ s., voir Jeammet, «Naissance et diffusion des Tanagréennes " dans Jeammet (éd.), 2003, p. 120-129.

20. Legs par disposition du 2 août 1914 de M. Alfred Hilarion Henri Baillehache-
Lamotte décédé le 15/02/1922. La figurine fut acceptée par le musée du Louvre le 6/12/1923 (Archives des Musées nationaux, dossier Z.8-1923) avec la mention "Grèce».

21. Une photographie des années 1950 nous montre un épiderme en effet plus préservé. La constitution de dossiers pour toutes les œuvres et de rapports de restauration est une pratique relativement récente pour les départements archéologiques du musée du Louvre.

22. Voir dans Jeammet (éd.), 2003 $\mathrm{n}^{\circ} 150$ (J. Becq), et Jeammet (dir.), 2010, $\mathrm{n}^{\circ} 207$, le croquis de M.-E. Meyohas. Analyses S. Colinart: C2RMF, dossier FZ30750, juin 2002.

23. Hasselin-Rous, Laugier, Martinez (dir.), 2009, p. 202-203, et cat. n ${ }^{\circ} 127$, p. 212. Don Bardac; dite trouvée à Priène. H. 28,3 cm. Besques, 1972, p. 126, D 847, pl. $155 \mathrm{a}$.

24. «Jaune recouvert de rose foncé pour les chairs et par-dessus préparation blanche recouverte de rose vif; rouge sur l'aile, les cheveux, le manteau avec repeint rouge sur la dorure; restes de dorure sur l'aile et le manteau ", op. cit., note supra, p. 212.

25. Des analyses sont également prévues sur cette œuvre.

26. De récentes études archéométriques du C2RMF (A. Bouquillon) ont ainsi permis d'attribuer de nombreuses figurines du Louvre aux ateliers tanagréens ou thébains, voir Jeammet (dir.), 2010, p. 111 sq., confortées par la récente découverte de la nécropole thébaine, voir V. Aravantinos, «Les fouilles des anciennes nécropoles dans la région de Thèbes » dans Jeammet, 2007, p. 59-66. Pour les analyses de Smyrne et sa région, voir Bouquillon dans Hasselin-Rous, Laugier, Martinez (dir.), 2009, p. 220-221.

27. L'indication fournie par le livre d'entrée est donc fiable. Voir Rumscheid, 2006, six figurines provenant de la maison 29 de Priène aujourd'hui conservées à Istanbul; la date d'acquisition par le Louvre est compatible: voir le résumé proposé par I. Hasselin-Rous dans le catalogue d'exposition 2009 (voir note 23). 
28. La Baillehache est en effet absolument intacte, ce qui est rarissime pour cette époque, où l'on constate le bris quasi systématique de figurines avant la déposition dans la tombe. Cet état prêche encore en faveur d'un lieu d'enfouissement domestique. L'état de conservation de l'Éros est moins satisfaisant (manques notamment au niveau des extrémités; cassures des membres). Cela peut s'expliquer par la chute de l'œuvre initialement suspendue, comme l'était certainement l'ensemble de ces Erotes. Le choix du matériau, le traitement en ronde-bosse, la présence des ailes et de trous au revers, comme la confrontation avec certains documents iconographiques et littéraires, parlent en ce sens, voir Rumscheid, 2006, p. 344-347.

\section{Bibliographie sélective}

Blume C., 2014, "Bright Pink, Blue and Other Preferences" dans Østergaard J. S., Nielsen A. M. (éds), 2014, p. 166189.

Bouquillon A., Colinart S., Porto E., Zink A., 2003, "Authenticité, matières et couleurs. Etude en laboratoire des Tanagréennes du Louvre» dans Jeammet (éd.), 2003, p. 298-301.

Bouquillon A., Zink A., Porto E., 2007, «Les Tanagras du Louvre à la lumière des analyses scientifiques. Authenticité, matières, provenances » dans Jeammet (éd.), 2007, p. 91-100.

Bourgeois B., 2014, «Thérapéia » dans Østergaard J. S., Nielsen A. M. (éds), 2014, p. 190-207.

Bourgeois B., 2007, «Pratiques artisanales de la couleur, Enquête sur la polychromie des Tanagréennes ", dans Jeammet, 2007, p. 81-89.

Bourgeois B., Jeammet V., Pagès-Camagna S., 2012-2013, « Color siderum. La dorure des terres cuites grecques aux époques hellénistique et romaine ", Bulletin de Correspondance Hellénique 136-137, p. $483-510$.
Bourgeois B., Jockey Ph., 2007, «Le marbre, l'or et la couleur. Nouveaux regards sur la polychromie de la sculpture hellénistique de Délos", dans Descamps-Lequime (dir.), 2007, p. 163191.

Bourgeois B., Jockey Ph., Karydas A., 2009, "New Researches on Polychrome Hellenistic Sculpture in Delos, III: the Gilding Processes. Observations and Meanings" dans Jockey Ph. (dir.), Leukos Lithos. Interdisciplinary Studies on Mediterranean Ancient Marble and Stones, Paris, p. 645-661.

Brecoulaki H., 2006, La peinture funéraire de Macédoine. Emplois et fonctions de la couleur IV $V^{e} I I^{e}$ av.J.-C., Centre de Recherches de l'Antiquité Grecque et Romaine, Fondation Nationale de la Recherche Scientifique, Meletimata 48 , Athènes.

Brecoulaki H., 2000, «Sur la techné de la peinture grecque ancienne d'après les monuments funéraires de Macédoine", BCH 124, 2000, p. 189-216.

Brinkmann V., Wünsche R. (éd.), 2003, Bunte Götter: die Farbigkeit antiker Skulptur, cat. exp. Munich, 2003.

Burr D., 1934, Terra-cottas from Myrina in the Museum of Fine Arts, Boston, Thèse Bryn Mawr College, Vienne, 1934.

Descamps-Lequime S. (dir.), 2007, Peinture et couleur dans le monde grec antique, Actes du colloque du musée du Louvre (10 et 27 mars 2004), Paris.

Higgins R., 1970, "The Polychrome Decoration of Greek Terracottas", Studies in Conservation 15, p. 272-277.

Hamdorf F. W. (éd.), 1996, Hauch des Prometheus. Meisterwerke in Ton, cat. exp. Staatliche Antikensammlungen München.

Hasselin-Rous I., Laugier L., Martinez J.-L. (dir.), 2009, D'Izmir à Smyrne. Découverte d'une cité antique, cat. exp. Musée du Louvre.

Jeammet V., 2014, "Sculpture en miniature», dans Østergaard J. S., Nielsen A. M. (éds), 2014, p. 208-221 (Phainoméride Myr 163, p. 222, cat. 56 p. 327).

Jeammet V. (éd.), 2003, Tanagra. Mythe et archéologie, cat. exp. Musée du Louvre, Montréal, Musée des Beaux Arts.

Jeammet V., 2007, «Un certain goût pour les Tanagras: du XIX ${ }^{\mathrm{e}}$ siècle à l'Antiquité ", dans Jeammet (éd.), p. 33-43.

Jeammet V. (éd.), 2007, Tanagras. De l'objet de collection à l'objet archéologique. Actes du colloque du musée du Louvre/ Bibliothèque nationale de France, (Paris, 22 nov. 2003), Paris.

Jeammet V. (dir.), 2010, Tanagras. Figurines for Life and Eternity, The Louvre's Collection of Figurines, cat. exp. Valencia, Fondation Bancaja, Valence.

Jeammet V., Knecht C., Pagès-Camagna S., 2007, «La couleur sur les terres cuites hellénistiques: les figurines de Tanagra et de Myrina dans la collection du musée du Louvre ", dans DescampsLequime (dir.), p. 193-203.

Laumonier A., 1956, Exploration archéologique de Délos. Les figurines de terre cuite, vol. 23, Athènes.

Mathieux, N., 2007, «Des tanagras à l'encan ", dans Jeammet (éd.), 2007, p. $45-57$.

$\varnothing$ stergaard J. S., Nielsen A. M. (éds), 2014, Transformations. Classical Sculpture in Colour, Ny Carlsberg Glyptotek, Copenhague.

Rayet O., 1878, «L'art grec au Trocadéro », Gazette des Beaux-Arts 18, p. 347-370.

Rouveret A., 2007, «La couleur retrouvée. Découvertes de Macédoine et textes antiques » dans Descamps-Lequime (dir.), 2007, p. 69-80.

Rumscheid F., 2006, Die figürlichen Terrakotten von Priene, Wiesbaden.

Zimmer G. (éd.), 1994, Bürgerwelten. Hellenistische Tonfiguren und Nachschöpfungen im 19.Jh., cat. exp. Berlin. 